SLAC-PUB-6418

January 1994

(T)

\title{
The Vacuum Theta-Angle is Zero in Non-Abelian Gauge Theories*
}

\author{
Valentin V. Khoze \\ Stanford Linear Accelerator Center \\ Stanford University, Stanford, California 94309
}

\begin{abstract}
We argue that gauge transformations with arbitrary fractional winding numbers should be allowed in a non-Abelian gauge theory. This eliminates the topological distinction between large and small gauge transformations. We prove that the states which satisfy the Gauss's law constraint are strictly invariant under gauge transformations, i.e. no phase shift occurs. In other words, the vacuum $\theta$-angle is zero.

Our observation does not resolve the strong CP problem.
\end{abstract}

Submitted to Physics Letters B

* Work supported by the Department of Energy, contract DE-AC03-76SF00515. 
It is usually assumed that the only gauge transformations allowed in Yang-Mills theory are those with integer winding numbers. This assumption is based on some topological argument, which we will discuss later, and on the properties of instantons. Instanton solutions describe tunneling processes only between vacua whose winding numbers differ by an integer. There exist, however, other classes of solutions in Minkowski space which correspond to classically allowed transitions between the configurations with arbitrary winding numbers [1]. If after such a transition we do not want to end up in a state missing from the classification based on integer winding numbers, we should expand this classification to include states with arbitrary fractional winding numbers.

In this note we will point out that the boundary conditions appropriate to this generalization imply that the physical states of the theory are invariant under all gauge transformations, and that, correspondingly, the vacuum $\theta$-angle is zero. This conclusion is more conceptual than practical; adding a term $i \bar{\theta} F \tilde{F}$ to the lagrangian produces observable CP-violating expectation values with the usual $\bar{\theta}$-dependence.

We first quantize the theory in the Hamiltonian formalism and then review the traditional argument which leads to the following gauge transformation law [2] for the physical states of the Hilbert space:

$$
\mathcal{G}^{U}|\Psi\rangle_{\text {Phys }}=e^{-i \nu[U] \theta}|\Psi\rangle_{\text {Phys }}
$$

Here $\nu[U]$ is the (integer) winding number of the gauge transformation $U$ and $\mathcal{G}^{U}$ is the operator in the Hilbert space which implements this gauge transformation. The physical states are those which obey the Gauss's law constraint. The $\theta$-angle is the same for all physical states and it is usually said that different values of $\theta$ describe different $\theta$-worlds. Each $\theta$-world can be viewed as a tower built on the corresponding $\theta$-vacuum.

Next we allow in the theory gauge transformations with arbitrary fractional winding numbers. This lifts the topological distinction between large $(\nu \neq 0)$ and small $(\nu=0)$ gauge transformations, so that all gauge transformations (large and small) can be continuously deformed into each other. Then we prove that under any gauge transformation a physical state transforms into itself,

$$
\mathcal{G}^{U}|\Psi\rangle_{\text {Phys }}=|\Psi\rangle_{\text {Phys }}
$$

This implies that the $\theta$-angle of eq. (1) is always equal to zero. 
We consider a theory with the action

$$
S=\frac{1}{2 g^{2}} \int d^{4} x \operatorname{tr}\left(F^{\mu \nu} F_{\mu \nu}\right)
$$

where the field strength is $F_{\mu \nu}=\partial_{\mu} A_{\nu}-\partial_{\nu} A_{\mu}+\left[A_{\mu}, A_{\nu}\right]$ and $A_{\mu}=g A_{\mu}^{a} \tau^{a} /(2 i)$ is the antihermitian vector-potential. To simplify our presentation we suppress the fermion fields and choose the number of colors to be two. The theory is invariant under local gauge transformations

$$
A_{\mu} \rightarrow A_{\mu}^{U}=U^{\dagger}\left(\partial_{\mu}+A_{\mu}\right) U
$$

where $U$ is an $S U(2)$ matrix.

From now on we choose the $A_{0}=0$ gauge. We write the equations of motion, $D^{\mu} F_{\mu \nu}=$ 0 in a non-covariant form,

$$
\begin{gathered}
\left(D_{i} E_{i}\right)^{a}=0, \\
\partial_{t} E_{i}^{a}=(D \times B)_{i}^{a},
\end{gathered}
$$

where $E_{i}^{a}=F_{0 i}^{a}$ and $B_{i}^{a}=-1 / 2 \epsilon^{i j k} F_{j k}^{a}$ stand for electric and magnetic fields and D is the covariant derivative in the adjoint representation, $\left(D_{i} M\right)^{a}=\partial_{i} M^{a}+g \epsilon^{a b c} A_{i}^{b} M^{c}$. The dynamical variable of the theory is $A_{i}^{a}$, the canonical momentum is $-E_{i}^{a}$ and the Hamiltonian is given by

$$
H=\frac{1}{2} \int d^{3} x\left(E_{i}^{a 2}+B_{i}^{a 2}\right)
$$

The canonical commutation relation,

$$
\left[E_{i}^{a}(\mathbf{x}), A_{j}^{b}(\mathbf{y})\right]=i \delta^{i j} \delta_{a b} \delta^{(3)}(\mathbf{x}-\mathbf{y})
$$

implies

$$
\begin{gathered}
\partial_{t} A_{i}^{a}=i\left[H, A_{i}^{a}\right]=-E_{i}^{a}, \\
\partial_{t} E_{i}^{a}=i\left[H, E_{i}^{a}\right]=(D \times B)_{i}^{a},
\end{gathered}
$$

which reproduces the Ampère law (5.b) as an operator equation. The Gauss law (5.a) should be implemented as a constraint on the physical states of the Hilbert space,

$$
\left(D_{i} E_{i}\right)^{a}|\Psi\rangle_{\mathrm{Phys}}=0
$$


A gauge transformation (4) with

$$
U(\mathbf{x})=\exp \left[\Lambda^{a}(\mathbf{x}) \tau^{a} /(2 i)\right]
$$

is represented in the Hilbert space by the operator

$$
\mathcal{G}^{U}=\exp \left[-i / g \int d^{3} x E_{i}^{a}\left(D_{i} \Lambda\right)^{a}\right] .
$$

Indeed, it is straightforward to check that the following equations

$$
\begin{gathered}
\mathcal{G}^{U} A_{i} \mathcal{G}^{U^{\dagger}}=U^{\dagger}\left(\partial_{i}+A_{i}\right) U, \\
\mathcal{G}^{U} E_{i} \mathcal{G}^{U^{\dagger}}=U^{\dagger} E_{i} U,
\end{gathered}
$$

with $U$ given by (10), are satisfied by $\mathcal{G}^{U}$ of eq. (11) purely on the algebraic level, without requiring any integrations by parts. We also note that eqs. (12) imply that $\left[\mathcal{G}^{U}, H\right]=0$.

Thus, eqs. (12) ensure that any gauge transformation which can be cast in the form (10) is generated by the operator (11). However, in the standard treatment [2], the socalled large gauge transformations cannot be implemented by the exponential operator (11). Let us review the argument of Refs. [2]. First, one assumes that as $\mathbf{x} \rightarrow \infty$, any gauge function $U(\mathbf{x})$ approaches a direction-independent matrix. Then one can compactify the three-space into a three-sphere, $S^{3}$, and $U$ provides a map, $U: S^{3} \rightarrow S U(2) \approx S^{3}$. Such maps fall into disjoint homotopy classes labeled by the integers, called the winding numbers $\nu$,

$$
\nu[U]=-\frac{1}{24 \pi^{2}} \int d^{3} x \epsilon^{i j k} \operatorname{tr}\left(\left(U^{\dagger} \partial_{i} U\right)\left(U^{\dagger} \partial_{j} U\right)\left(U^{\dagger} \partial_{k} U\right)\right) .
$$

Gauge functions belonging to different classes cannot be deformed continuously into each other without violating the compactification assumption. In particular, only those in the zero winding number class are deformable to the identity and can be generated by the exponential operator (11). Such gauge transformations are called small and they can be written in the form (10) with $\Lambda^{a}(\mathbf{x}) \rightarrow 0$ at spatial infinity. For small gauge transformations the right hand side of eq. (11) can be integrated by parts and the Gauss's law constraint (9) requires

$$
\mathcal{G}^{U_{\text {SMALL }}}|\Psi\rangle_{\text {Phys }}=|\Psi\rangle_{\text {Phys }}
$$


But homotopically non-trivial gauge transformations, according to Refs. [2], are only phase-invariant,

$$
\mathcal{G}^{U}|\Psi\rangle_{\text {Phys }}=e^{-i \nu[U] \theta}|\Psi\rangle_{\text {Phys }} .
$$

In this treatment $\theta$ is not constructed, but only allowed by the disjoint nature of gauge transformations.

We may choose not to impose the compactification assumption and to allow $U$ to depend on the polar angles at spatial infinity. There is no difficulty with the boundary conditions; we can, for example, impose MIT bag [3] boundary conditions, which contain only $F_{\mu \nu}$ and not $A_{\mu}$ on the sphere of large radius. In this case, the above topological classification fails and all gauge functions can be continuously deformed into each other and are generated by the exponential operator (11). As an example of this statement we can consider a gauge function $U$ given by eq. (10) with

$$
\Lambda^{a}(\mathbf{x})=q \Omega(r) \hat{x}^{a},
$$

where $q$ is an arbitrary number, $\hat{x}$ is the unit three-vector in the radial direction and

$$
\left.\Omega(r)\right|_{r \rightarrow 0} \rightarrow 0,\left.\quad \Omega(r)\right|_{r \rightarrow \infty} \rightarrow 2 \pi .
$$

The winding number (13) of this gauge function is

$$
\nu[U]=q-\frac{\sin (2 \pi q)}{2 \pi} .
$$

Gauge functions of this form can be viewed as a continuous path of gauge functions labeled by a parameter $q$. This path connects a gauge function of winding number zero at $q=0$ with a gauge function of winding number one at $q=1$. The path interpolates through the gauge functions with fractional winding numbers at fractional values of $q$. The operator generating these gauge transformations is given by eq. (11) with $\Lambda$ and $\Omega$ from eqs. (16)(17).

Equation (18) illustrates that the definition (13) of the winding number is somewhat arbitrary. Indeed one might pick $\nu[U]$ to be any functional of $U$ which coincides with the right hand side of eq. (13) when $U$ is restricted to a direction-independent matrix at spatial infinity. 
Furthermore we note that eq. (15) with $\nu$ defined by eq. (13) is somewhat subtle for gauge transformations of this more general type. Indeed equation (18) implies that for $U_{1}$ and $U_{2}$ from the class (10), (16)-(17) with fractional $q_{1}$ and $q_{2}$,

$$
\nu\left[U_{1} U_{2}\right] \neq \nu\left[U_{1}\right]+\nu\left[U_{2}\right]
$$

and eq. (15) fails to represent the group. Of course, one may think that what we have here is a group representation up to a phase or projective representation:

$$
\begin{gathered}
\mathcal{G}^{U_{1}} \mathcal{G}^{U_{2}}|\Psi\rangle_{\text {Phys }}= \\
\exp \left[i \theta /(2 \pi)\left\{\sin \left(2 \pi\left(q_{1}+q_{2}\right)\right)-\sin \left(2 \pi q_{1}\right)-\sin \left(2 \pi q_{2}\right)\right\}\right] \mathcal{G}^{U_{1} U_{2}}|\Psi\rangle_{\text {Phys }}
\end{gathered}
$$

with

$$
\mathcal{G}^{U_{j}}=\exp \left[-i q_{j} / g \int d^{3} x E_{i}^{a}\left(D_{i} \Omega(r) \hat{x}\right)^{a}\right] .
$$

But now using eq. (21) we can immediately see that

$$
\mathcal{G}^{U_{1}} \mathcal{G}^{U_{2}}=\exp \left[-i\left(q_{1}+q_{2}\right) / g \int d^{3} x E_{i}^{a}\left(D_{i} \Omega(r) \hat{x}\right)^{a}\right] \equiv \mathcal{G}^{U_{1} U_{2}}
$$

which is in a contradiction with eq. (20). This demonstrates that eq. (15) with $\nu$ defined by eq. (13) is not a valid statement for gauge transformations of the type (10), (16)-(17). Even more generally, eq. (15) fails for any gauge transformation which can be transformed to a gauge transformation of the class (10), (16)-(17) making a small gauge transformation.

The only way to have no phase in (20) is to appropriately modify the definition of the winding number (13) for fractional values of $\nu$, that is we must choose $\nu=q$ instead of (13), and the gauge transformation law (15) then takes the form,

$$
\mathcal{G}^{U(q)}|\Psi\rangle_{\text {Phys }}=e^{-i q \theta}|\Psi\rangle_{\text {Phys }} .
$$

For integer values of $q$, eqs. (22) and (15) certainly agree.

Now we will prove that the $\theta$-angle on the right hand side of eq. (22) is zero. The left hand side of equation (22) should be viewed as an expansion in powers of $q$ and when we equate the terms proportional to the $q^{1}$ on the right and on the left we obtain,

$$
\theta|\Psi\rangle_{\mathrm{Phys}}=1 / g \int d^{3} x E_{i}^{a}\left(D_{i} \Omega(r) \hat{x}\right)^{a}|\Psi\rangle_{\mathrm{Phys}}
$$




$$
\begin{gathered}
=1 / g \int d^{3} x\left(\partial_{i}\left(E_{i}^{a} \Omega(r) \hat{x}^{a}\right)-\Omega(r) \hat{x}^{a}\left(D_{i} E_{i}\right)^{a}\right)|\Psi\rangle_{\text {Phys }} \\
=2 \pi / g \int d^{3} x \partial_{i}\left(\hat{x}^{a} E_{i}^{a}\right)|\Psi\rangle_{\text {Phys }}
\end{gathered}
$$

where the Gauss's law constraint (9) was used. We note that if we have restricted ourselves to MIT bag boundary conditions [3], this would imply that the component of $E_{i}^{a}$ normal to the boundary is zero at the boundary, and the right hand side of eq. (23) is zero. This implies that $\theta$ is zero for MIT bag boundary conditions. We will demonstrate that this is also true in general. Let us gauge transform the left and the right hand side of eq. (23) with the gauge function $V$ of the form,

$$
V(\mathbf{x})=\exp \left[\Phi(r) n^{a} \tau^{a} /(2 i)\right]
$$

where $\Phi$ is some function of $r$ and $n^{a}$ is an arbitrary $x$-independent unit-vector. The gauge transformation of eq. (23) reads,

$$
\theta \mathcal{G}^{V}|\Psi\rangle_{\text {Phys }}=1 / g \int d^{3} x \partial_{i}\left(\Omega(r) \hat{x}^{a} \mathcal{G}^{V} E_{i}^{a} \mathcal{G}^{V^{\dagger}}\right) \mathcal{G}^{V}|\Psi\rangle_{\text {Phys }}
$$

Under the action of $\mathcal{G}^{V}$ the state $|\Psi\rangle_{\text {Phys }}$ can at most acquire a phase which we cancel on the left and on the right of the above equation and arrive at the following expression,

$$
\theta|\Psi\rangle_{\mathrm{Phys}}=1 / g \int d^{3} x \partial_{i}\left(\Omega(r) \hat{x}^{a} \mathcal{G}^{V} E_{i}^{a} \mathcal{G}^{V^{\dagger}}\right)|\Psi\rangle_{\mathrm{Phys}}
$$

where the gauge transformation of the electric field reads in this case,

$$
\mathcal{G}^{V} E_{i}^{a} \mathcal{G}^{V^{\dagger}}=E_{i}^{a} \cos (\Phi)+\epsilon^{a b c} E_{i}^{b} n^{c} \sin (\Phi)+2 n^{a} n^{b} E_{i}^{b} \sin ^{2}(\Phi / 2)
$$

Equation (25) should hold for any $V$ of the form (24), in other words the left hand side of eq. (25) is independent of asymptotic value of $\Phi$ and on the choice of $n^{a}$. Let us choose $\Phi$ such that $\left.\Phi\right|_{r \rightarrow \infty} \rightarrow \pi / 2+\alpha$, differentiate both sides of eq. (26) with respect to $\alpha$ and then set $\alpha$ equal to zero. This establishes the following equality:

$$
2 \pi / g \int d^{3} x \partial_{i}\left(\hat{x}^{a} E_{i}^{a}-\hat{x}^{a} n^{a} E_{i}^{b} n^{b}\right)|\Psi\rangle_{\mathrm{Phys}}=0
$$

which is possible only if

$$
\int d^{3} x \partial_{i}\left(\hat{x}^{a} E_{i}^{a}\right)|\Psi\rangle_{\mathrm{Phys}}=0 .
$$


This proves that $\theta=0$ and that the transformation law

$$
\mathcal{G}^{U}|\Psi\rangle_{\text {Phys }}=|\Psi\rangle_{\text {Phys }}
$$

holds for arbitrary gauge transformations.

We mention that the derivation of eq. (28) is based on the fact that $E_{i}^{a}$ is a gaugevariant operator. Thus our conclusion, eq. (29) is not necessarily true in Abelian models, see Ref. [4].

The gauge transformation law (29) implies that the so-called theta-vacua do not contain any theta and thus the vacuum is unique. It is given just by the integral over the whole gauge orbit of the perturbative vacuum. We note again that when fractional winding numbers are allowed there is just one gauge orbit, that is all gauge transformations can be continuously deformed to each other.

It is worthwhile mentioning that the $\theta F \tilde{F}$ term can be obtained in the functional integral formalism starting from the $\theta$-independent classical action, eq. (3), and the $\theta$ dependent gauge transformation, eq. (15) [2]. On the other hand we have just argued that $\theta=0$ in eq. (15). This observation, however, does not prove that the $\theta F \tilde{F}$ term is zero. In fact, one can just start with the action which contained $\bar{\theta} F \tilde{F}$. The canonical momentum, $\Pi_{i}^{a}$, of this theory is now $\bar{\theta}$-dependent. The gauge transformation operator, however, remains (11) with $E_{i}^{a}$ and not $-\Pi_{i}^{a}$ in the exponent. This demonstrates that $\bar{\theta}$

cannot be removed from the formalism. Thus the $\bar{\theta}$-strong $\mathrm{CP}$ parameter does not seem to have much in common with the $\theta$-vacuum-angle which we proved is zero.

\section{Acknowledgments}

We are very grateful to Michael Peskin, Helen Quinn and Marvin Weinstein for most valuable discussions and comments. We also thank Arnd Brandenburg, Edward Farhi, Alex Kagan, Eric Sather and Peter van Driel for useful conversations and comments on the manuscript. 


\section{References}

[1] E. Farhi, V.V. Khoze and R. Singleton, Phys. Rev. D 47, 5551 (1993).

[2] R. Jackiw and C. Rebbi, Phys. Rev. Lett. 37, 172 (1976);

C. Callan, R. Dashen and D. Gross, Phys. Lett. 63 B, 334 (1976).

[3] A. Chodos, R.L. Jaffe, K. Johnson and V.F. Weisskopf, Phys. Rev. D 12, 3471 (1974).

[4] H.R. Quinn and M. Weinstein, Phys. Rev. D 17, 1063 (1978). 